\title{
Sister Chromatid Cohesion and Aneuploidy
}

\author{
Erwan Watrin* and Claude Prigent ${ }^{*}$ \\ Research Institute of Genetics and Development \\ Centre National de la Recherche Scientifique \\ University of Rennes I
}

France

\section{Introduction}

In all living organisms, cells arise from the division of a pre-existing cell and inherit one copy of the genome identical to that of the mother cell. Any defect in chromosome segregation can lead to aneuploidy via chromosome gain or loss, and consequently to genetic instability, thereby paving the way for malignant growth and cancer. Thus, the faithful distribution of one copy of each chromosome between the two daughter cells is essential to maintain genome stability. Cells have evolved elaborate control mechanisms to ensure that chromosome segregation occurs in an accurate manner. From yeast to human, replicated DNA molecules are held together from their synthesis during S-phase until their separation in mitosis. This sister chromatid cohesion is absolutely essential for faithful transmission of replicated chromosomes during mitosis (for review, see Nasmyth, 2001). Indeed, in early mitosis, cohesion between sister chromatids counteracts the pulling forces exerted by the microtubule filaments emanating from the opposite poles of the mitotic spindle, thereby allowing the proper alignment of chromosomes on the metaphase plate, and their faithful segregation during the subsequent anaphase. In the case of a defective cohesion, the sister chromatids would float around in the cellular space and, as a consequence, would fail to be equally distributed between the two future daughter cells. Cohesion between sister chromatid is not only required for the proper distribution of replicated genomes during mitosis, but is also essential in postreplicative interphase cells for the repair of DNA double-strand breaks by homologous recombination (Sjogren and Nasmyth, 2001)(for review, see Strom and Sjogren, 2007; Watrin and Peters, 2006). The homologous recombination pathway uses an identical copy of the damaged DNA fibre, typically the sister chromatid, as a template to faithfully repair the altered DNA. For the homologous recombination machinery to process chromosome breaks, the broken chromatid and the sister chromatid that is used as a template have to be positioned and maintained in close proximity. It is believed that sister chromatid cohesion provides this spatial proximity between damaged and template chromatids. Consistently, proteins that are required for sister chromatid cohesion are also required for the repair of DNA doublestrand breaks in postreplicative cells (for review, see Watrin and Peters, 2006). Therefore, sister chromatid cohesion plays an essential role in restoring and maintaining the integrity of the genome upon genotoxic stress.

* Corresponding Author 
In all eukaryotes studied so far, sister chromatid cohesion depends on a multi-subunit complex called cohesin (Losada et al., 1998; Strunnikov et al., 1993; Toth et al., 1999). This complex forms a ring-like structure, and it has been proposed that cohesin entraps replicated DNA molecules in this proteinaceous ring (Gruber et al., 2003; Haering et al., 2002). In addition to its role in sister chromatid cohesion, the cohesin complex has also been shown to be involved in other aspects of chromosome biology, which include the control of gene expression and the regulation of the DNA damage checkpoint that will not be further discussed here.

Over the last decade, we began to understand at the molecular level how cohesin functions in sister chromatid cohesion and how the cohesion apparatus is regulated during the cell cycle. In addition, accumulating evidence in human and animal models highlighted the importance of the cohesion apparatus in protecting somatic cells from aneuploidy and cancer, and germ cells from improper meiotic segregation that leads to aneuploid gametes and infertility. Here we present an overview of the current knowledge on the sister chromatid cohesion apparatus, its molecular actors and regulatory mechanisms in human. In addition we will discuss a defective cohesion as a cause for aneuploidy in somatic and germinal cells, and its involvement in human pathologies. Finally we will try to identify future directions that we need to explore to better understand the basis of aneuploidy-driven genomic instability.

\section{Composition and architecture of the cohesin complex}

\subsection{The cohesin core complex}

The cohesin core complex consists of four subunits (Figure 1). The two core subunits Smc1 and Smc3 belong to the structural maintenance of chromosome (SMC) protein family. SMC proteins fold back on themselves creating a long intramolecular anti-parallel coiled-coil that separates a "hinge" domain and a globular ATPase head which is formed by the reunion of the C- and N-terminal regions (Melby et al., 1998; Michaelis et al., 1997; Strunnikov et al., 1993). Within the cohesin complex, Smc1 and Smc3 bind tightly to each other via their hinge domain, and form a V-shaped heterodimer. In somatic cells, the ATPase domains of Smc1 and Smc3 are physically linked to each other by the kleisin subunit Scc1 (also called Rad21 or Mcd1). Scc1 subunit associates by its $\mathrm{N}$ - and C-termini to the ATPase heads of Smc3 and Smc1, respectively (Haering et al., 2002; Haering et al., 2004). The reunion of the Smc1/Smc3 heterodimer together with the Scc1 protein forms a tripartite molecular ring with an inner diameter of $40 \mathrm{~nm}$ (Figure 1) (Haering et al., 2002). This ring structure has been observed by electron microscopy imaging of cohesin complexes isolated from human cells as well as from Xenopus laevis interphase egg extract (Anderson et al., 2002). The cohesin ring is large enough to accommodate two 10-nanometer DNA fibres, and it has been proposed that cohesin maintains the cohesion between replicated chromatids by embracing them within its ring (Gruber et al., 2003; Haering et al., 2002). According to the widely accepted ring model (Figure 1), cohesin complex mediates cohesion between replicated DNA fibres simply by topological links. Alternative ring-based models have also been proposed for cohesinmediated sister chromatid cohesion, like the "handcuff" model, where each sister chromatid of a pair would be encircled within distinct cohesin complexes that would interact with each others and may thereby physically connect sister chromatids (Zhang et al., 2008b). Also, it has been proposed that two cohesin complexes would oligomerise into a larger ring around DNA (Huang et al., 2005). Besides interacting with Smc1 and Smc3, Scc1 protein also binds 
to the fourth cohesin subunit called Scc3 in yeast, of which two orthologs exist in vertebrates, called stromalin antigen 1 and 2 (SA1 and SA2). Within the cohesin complex, SA1 and SA2 interact with Scc1 in a mutually exclusive manner (Losada et al., 2000; Sumara et al., 2000), implying that two cohesin core complexes, referred to as cohesin ${ }^{\mathrm{SA} 1}$ and cohesin $^{\mathrm{SA} 2}$, coexist in vertebrates somatic cells. It has recently been suggested that cohesin complexes containing SA1 or SA2 have specialised function in sister chromatid cohesion at telomeres and at centromeres, respectively (Canudas and Smith, 2009).

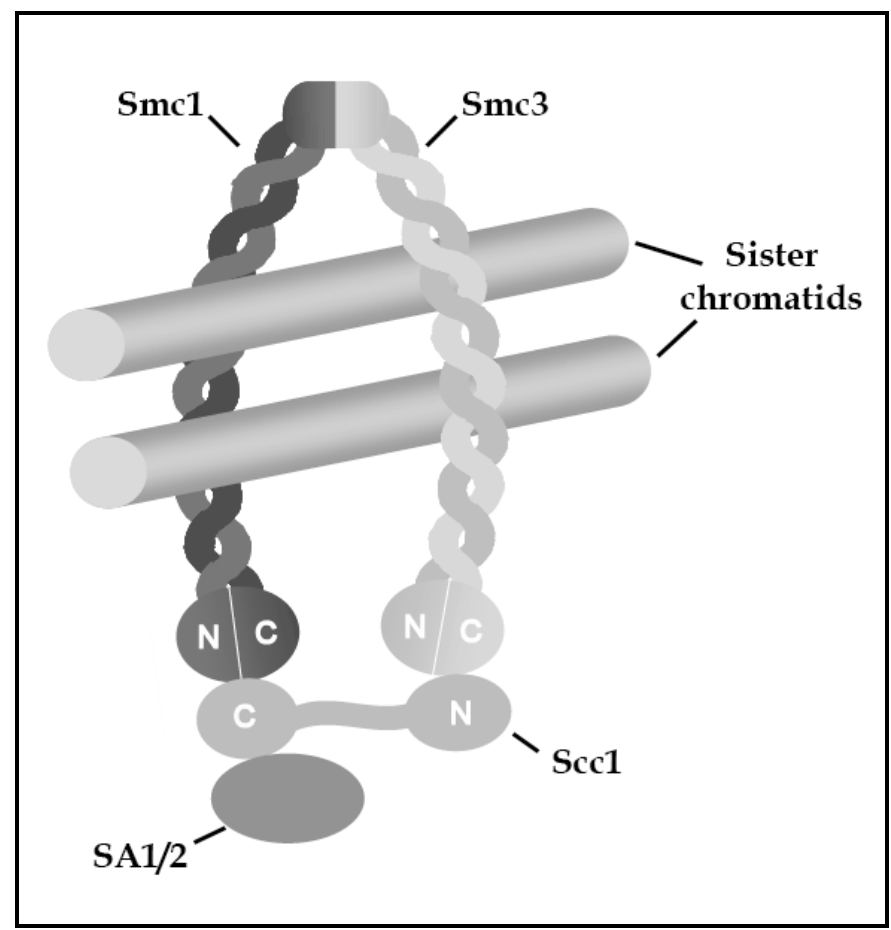

The vertebrate cohesin core complex consists of the four subunits Smc1, Smc3, Scc1 and SA1 or SA2. The association of Smc1, Smc3 and Scc1 forms a tripartite molecular ring. According to the ring model (Gruber et al., 2003; Haering et al., 2002), cohesin maintains sister chromatid cohesion by embracing the replicated chromatids within its ring. Cohesion is therefore mediated solely by physical, topological linkages.

Fig. 1. Composition and architecture of the cohesin core complex and the ring model

\subsection{Cohesin associated factors}

Besides the four core cohesin subunits, additional proteins have also been shown to be part of the cohesin complex. These evolutionary conserved proteins are named Pds5, Wapl and Sororin. In vertebrates, two homologs of Pds5 exist, called Pds5A and Pds5B, which are both able to associate with either cohesinSA1 or cohesin ${ }^{\mathrm{SA} 2}$ (Losada et al., 2000; Sumara et al., 2000). Therefore four distinct cohesin complexes are present in vertebrates cells. From yeast to fly, Pds5 is essential for the proper cohesion between sister chromatids (Dorsett et al., 2005; 
Hartman et al., 2000; Panizza et al., 2000; Tanaka et al., 2001; Wang et al., 2002. In vertebrate cells, however, depletion of Pds5A or Pds5B does not result in strong sister chromatid cohesion defects (Losada, 2005). Quite the opposite, it has recently been shown that depletion of both Pds5A and Pds5B from Xenopus laevis egg extract does not impair sister chromatid cohesion, but instead, severely affects cohesin dissociation and cohesion loss from chromosome arms in mitosis (Shintomi and Hirano, 2009), suggesting that Pds5A and Pds5B function in the dissociation of cohesin complexes from chromosome arms and thereby in the resolution, i.e. the physical separation, of the sister chromatids in early mitosis (see below).

Vertebrate cohesin also binds to Wapl (Gandhi et al., 2006; Kueng et al., 2006), a protein that was first identified in Drosophila, where it has been involved in the establishment of heterochromatin (Verni et al., 2000). Wapl homologs also exist in yeasts (called Rad61 or Wpl1) where it also associates with the cohesin complex (Rolef Ben-Shahar et al., 2008). It has been shown in human that the interaction of Wapl with cohesin depends on the SA1/SA2 and Scc1 subunits (Kueng et al., 2006). Furthermore, Wapl interacts with Pds5 proteins (Shintomi and Hirano, 2009) through a conserved motif that consists of the triplet sequence phenylalanine-glycin-phenylalanine residues (called the FGF motif). In vertebrates, Wapl is dispensable for sister chromatid cohesion, but plays an essential role in the removal of cohesin complexes from chromosome arms during early mitosis, similarly to Pds5 proteins (see below).

Finally, a small protein called Sororin also interacts with cohesin complexes (Rankin et al., 2005; Schmitz et al., 2007). Like the core cohesin complex, Sororin has been shown to be essential for cohesion between sister chromatids in vertebrates (Rankin et al., 2005; Schmitz et al., 2007), but is dispensable for cohesin's ability to associate with chromatin (Schmitz et al., 2007). Recent work has shown that the function of Sororin in sister chromatid cohesin depends on its ability to compete with Wapl for its binding to Pds5 proteins via its FGF motif (Nishiyama et al., 2010), strongly suggesting that Sororin acts in cohesion by antagonising Wapl's capacity to dissociate cohesin complexes from the chromatin (see below).

\subsection{Composition of cohesin in meiosis}

Similar to mitosis, faithful segregation of homologous chromosomes and sister chromatids during meiosis also depends on sister chromatid cohesion and on cohesin complexes (see below). However, the composition of the cohesin complex in meiotic cells slightly differs from that of somatic cells (for review, see Petronczki et al., 2003). The subunit Scc1 is replaced by a meiosis-specific variant called $\operatorname{Rec} 8$, and the Smc1 $\beta$ isoform can substitute for the canonical Smc1 (also referred to as Smc1a). Finally, SA1 and SA2 proteins can both be replaced by the meiotic form STAG3. Therefore, meiotic cells contain different forms of cohesin complexes, specific functions of which are still largely unknown.

\section{Regulating cohesin and sister chromatid cohesion}

In vertebrate cells, cohesin is loaded onto chromatin from telophase to early G1 phase. During S phase, sister chromatid cohesion is established, and maintained throughout G2 phase. Finally, cohesin complexes are removed from chromosomes during mitosis to allow physical separation of sister chromatids and faithful segregation of replicated chromosomes during anaphase (Figure 2). 


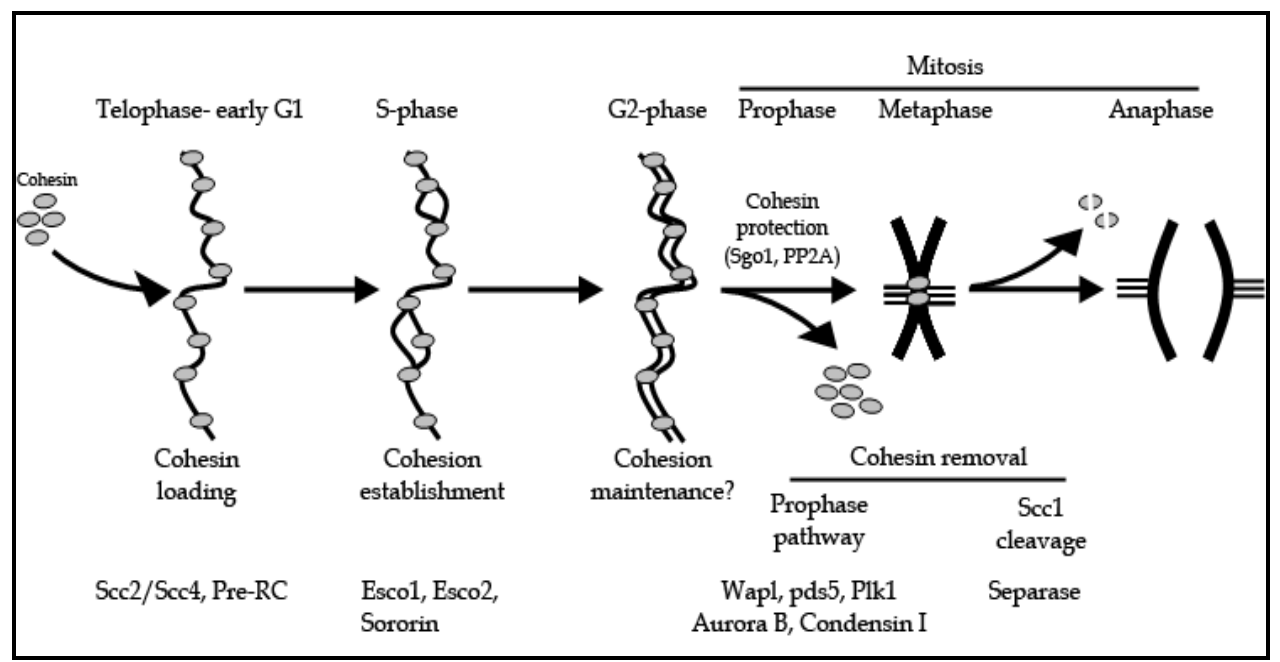

Cohesin loading onto chromatin occurs from telophase to early G1 and depends on the loading complex Scc2/Scc4. In addition, this process also requires that pre-RC complexes have assembled on chromatin. During $S$ phase, cohesion between duplicated sister chromatids is established. This process depends on the activity of the two acetyltransferases Esco1 and Esco2. The protein sororin is also essential for cohesion establishment. Sister chromatid cohesion is maintained during G2, although it is still unclear whether cohesion maintenance requires specific factors. Cohesin is removed from chromosomes during early mitosis in a two-step manner. During prophase, cohesin complexes dissociate from chromosome arms in a process that depends on the activity of the mitotic kinases Plk1 and Aurora B, the association of condensin I with chromosomes, as well as on Wapl and Pds5 proteins. Centromeric cohesin is protected from removal by the prophase pathway by Sgo1 and PP2A. At the metaphase-to-anaphase transition, the protease separase is activated and cleaves centromeric cohesin complexes. Complete removal of cohesin from chromosomes allows segregation of sister chromatids.

Fig. 2. Regulation of sister chromatid cohesion during cell cycle in human

\subsection{Loading of cohesin onto chromatin}

In all eukaryotes studied so far, loading of cohesin onto chromatin depends on an additional complex formed by two proteins, called Scc2 and Scc4 (Bernard et al., 2006; Ciosk et al., 2000; Furuya et al., 1998; Gillespie and Hirano, 2004; Rollins et al., 2004; Takahashi et al., 2004; Watrin et al., 2006). Furthermore, cohesin loading onto chromatin in Xenopus egg extract also requires that the pre-replication complex (pre-RC) assembles on chromatin during the exit from mitosis (Gillespie and Hirano, 2004; Takahashi et al., 2004). It has also been show that the ATPase activity of the Smc1/Smc3 heterodimer is essential for cohesin to bind to chromatin (Arumugam et al., 2003; Weitzer et al., 2003). How the chromatin fibre can enter the cohesin ring has been a long-lasting question in the field. Biochemical approaches combined with genetics studies have provided insights into molecular events that govern this process, which have been reviewed elsewhere (for review, see Nasmyth and Haering, 2009; Peters et al., 2008). Therefore, we will present here only an overview of key findings. The cohesin ring must be opened to allow the entry of the DNA therein. It has been proposed that Smc1 and Smc3 transiently dissociate at the hinge domain, thereby allowing 
DNA to enter into the cohesin ring (Gruber et al., 2006; Milutinovich et al., 2007). Given that in S. cerevisiae, the hinge domain of Smc1/Smc3 is able to interact with their ATPase heads (Mc Intyre et al., 2007), one can envision that ATP hydrolysis by Smc1/Smc3 ATPase heads triggers a conformational change of the cohesin complex, which would in fine result in the transient opening of the hinge and in the entry of DNA into the cohesin ring.

\subsection{Establishment of sister chromatid cohesion}

The establishment of cohesion between sister chromatids takes place while DNA is replicated during S phase (Uhlmann and Nasmyth, 1998) and depends on the activity of an acetyltransferase called Eco1/Ctf7 in yeast (Skibbens et al., 1999; Toth et al., 1999). Eco1 becomes dispensable once replication has been achieved, indicating that the cohesion can only be established during replication, i.e. when sister chromatids are synthesised. However, it has to be noticed that cohesion can be established de novo in postreplicative cells, but only upon DNA double-strand break (Kim et al., ; Strom et al., 2007; Unal et al., 2007). It has recently been shown that in yeast, Eco1 acetylates two lysine residues in the ATPase region of Smc3, and that these acetylations are essential for the establishment of sister chromatid cohesion (Rolef Ben-Shahar et al., 2008; Unal et al., 2008; Zhang et al., 2008a). In vertebrate cells, two orthologs of Eco1 exist, called Esco1 and Esco2 (Hou and Zou, 2005). Similar to the situation in yeast, Smc3 becomes acetylated during replication at two evolutionary conserved lysine residues, at position 105 and 106 (Zhang et al., 2008a). Simultaneous inactivation in human cells of Esco1 and Esco2 impairs the acetylation of Smc3 whereas depletion of either Esco1 or Esco2 has little impact, if any, on Smc3 acetylation (Nishiyama et al., 2010; Zhang et al., 2008a), indicating that these two acetyltransferases are at least partly redundant for their function in Smc3 acetylation. Interestingly, acetylation of Smc3 becomes dispensable for sister chromatid cohesion when either Wapl or Pds5 are inactivated (Feytout et al., 2011; Rowland et al., 2009; Sutani et al., 2009). This is consistent with the function of Wapl and Pds5 in destabilising sister chromatid cohesion. Recently, it has further been shown that the acetylation of Smc3 triggers a rearrangement in cohesin subunit interactions (Nishiyama et al., 2010). Indeed, Smc3 acetylation promotes binding of Sororin to cohesin, where Sororin displaces Wapl from its interaction with Pds5 proteins. The authors further showed that Sororin also contains an FGF motif that competes with that of Wapl for its binding to Pds5. Therefore the establishment of sister chromatid cohesion depends on the protein Sororin that might counteracts Wapl's ability to dissociate cohesin complexes from chromatin (Nishiyama et al., 2010). In human cultured cells, fluorescence recovery after photobleaching (FRAP) experiments have shown that the binding of cohesin complexes to chromatin is very dynamic in G1 phase cells, and that the binding of half of chromatin-bound cohesin pool becomes very stably associated with DNA in G2 phase cells (Gerlich et al., 2006). It has been proposed that this stably-bound pool of cohesin is responsible for sister chromatid cohesion (Gerlich et al., 2006). Consistent with this possibility, the inactivation of Sororin results in the reduction of the stably-bound pool of cohesin (Schmitz et al., 2007). Conversely, inactivating Wapl induces an increase in the stable pool of chromatin-bound cohesin (Kueng et al., 2006).

Altogether, these data are in favour of a model, in which the acetylation of Smc3 during replication triggers the recruitment of Sororin, the consequent displacement of the cohesiondestabilising protein Wapl, ultimately leading to the creation of a cohesive state of cohesin complexes and to the establishment of sister chromatid cohesion. 


\subsection{Removal of cohesin from chromosomes during mitosis}

Cohesion between sister chromatid is maintained during G2 until mitosis, when it must be dissolved to allow the physical separation of the two sets of replicated chromosomes. Unlike in yeasts, vertebrate cohesin is removed from chromosomes in a two-step manner from the onset of mitosis until the metaphase-to-anaphase transition (Figure 2).

\subsubsection{The prophase pathway}

In vertebrates, the bulk of cohesin complexes ( 90 - $95 \%$ ) is removed from the chromosome arms during prophase and prometaphase (Sumara et al., 2000; Waizenegger et al., 2000), whereas some cohesin complexes remain bound to chromosomes at centromeric regions (Waizenegger et al., 2000). This first wave of cohesin removal from chromosomes is referred to as the prophase pathway, which depends on several factors that include the mitotic kinases Aurora B (Dai et al., 2006; Losada et al., 2002) and Plk1 (Lenart et al., 2007; Losada et al., 2002; Sumara et al., 2002) and the proteins Wapl (Gandhi et al., 2006; Kueng et al., 2006) and Pds5 (Shintomi and Hirano, 2009). Plk1 is thought to promote cohesin release from chromatin by phosphorylating the SA2 subunit (Losada et al., 2002; Sumara et al., 2002). Consistent with this possibility, the expression of a mutated version of SA2 that can no longer be phosphorylated in mitosis triggers a persistent binding of cohesin complexes on chromosome arms in prometaphase (Hauf et al., 2005). This strongly suggests that Plk1 participates in the dissociation of cohesin from the chromosomes by directly phosphorylating the SA2 subunit. In addition, it has also been shown that the cohesinrelated complex Condensin I is required for cohesin removal from chromosome arms (Hirota et al., 2004), although the role of Condensin I in the prophase pathway remains unclear.

Central actors to the removal of cohesin by the prophase pathway are Wapl and Pds5 proteins (Gandhi et al., 2006; Kueng et al., 2006; Shintomi and Hirano, 2009). Consistently, inactivation of either Wapl or Pds5 results in the persistence of cohesin complexes along the chromosome arms, which leads to unresolved sister chromatids in prometaphase (Gandhi et al., 2006; Kueng et al., 2006; Shintomi and Hirano, 2009). Recently, it was shown that sororin, which promotes sister chromatid cohesion by counteracting cohesindestabilising activity of Wapl (see above), is phosphorylated in mitosis (Nishiyama et al., 2010). Sororin phosphorylation correlates with the loss of its ability to displace Wapl from Pds5 in vitro. Therefore, it has been proposed that the mitotic phosphorylation of Sororin inactivates its ability to displace Wapl from binding to Pds5, which in turn leads to the association of Wapl to Pds5 and the dissociation of cohesin complexes from chromosomes (Nishiyama et al., 2010).

Whereas most of cohesin complexes are removed from the chromosome arms during prophase, the cohesin complexes located at centromeric regions are protected from the prophase pathway. This protection of centromeric cohesion is essential to maintain sister chromatid cohesion until anaphase onset, and allows the proper bipolar attachment of mitotic chromosomes on the metaphase plate. At this stage of mitosis, sister chromatids are held together only at centromeres, giving rise to the classical X-shaped mitotic chromosomes. Cohesin protection at centromeres depends on a protein called Shugoshin (guardian spirit in Japanese) also known as Sgo1 (Kitajima et al., 2004; McGuinness et al., 
2005; Salic et al., 2004). Sgo1 interacts with the protein phosphatase PP2A and both proteins are enriched at centromeres in early mitosis. It is believed that Sgo1 recruits PP2A to the centromeres, where PP2A dephosphorylates the cohesin subunit SA2, thereby counteracting the dissociation activity of the kinase Plk1 (Kitajima et al., 2006). The inhibition of the kinase Aurora B has been shown to trigger abnormal localisation of Sgo1 along chromosome arms (Dai et al., 2006). This indicates that Aurora B is required for the proper enrichment of Sgo1 at centromeres, and might account for its role in the prophase pathway. Finally other factors have also been shown to be important for the protection of cohesin complexes at centromeres, including the mitotic kinases Haspin (Dai et al., 2006) and Bub1 (Tang et al., 2004). Recently, it has been shown that Bub1 kinase is required for the phosphorylation of the centromeric histone H2A at threonine 120 (T120), for Sgo1 enrichment at centromeres and for sister chromatid cohesion (Kawashima et al., 2010), suggesting that Bub1-mediated phosphorylation of H2A-T120 recruits Sgo1 and thereby participates in the protection of centromeric cohesion. Additional experimental works will be required to substantiate this view, and, more importantly, to obtain an integrated view of the molecular actors and mechanisms involved in the protection of sister chromatid cohesion at centromeres.

\subsubsection{Cleavage of cohesin by separase}

During early mitosis, sister chromatids remain tightly associated at centromeric regions until all the chromosomes have been properly bi-oriented on the mitotic spindle (Waizenegger et al., 2000). As long as all chromosomes have not been correctly aligned on the metaphase plate, a surveillance mechanism, called the spindle assembly checkpoint (SAC), inhibits the activity of the anaphase promoting complex, or cyclosome, (APC/c) thereby preventing the activation of separase and the inactivation of cyclin-dependent kinase 1 (Cdk1). Once all chromosomes have been bi-oriented on the mitotic spindle, the $\mathrm{SAC}$ is satisfied and, consequently, the inhibition of the APC/c is relieved. Activated APC/c can then ubiquitinate the separase inhibitory protein securin as well as the Cdk1 activator cyclin B, leading to their degradation by the $26 \mathrm{~S}$ proteasome (reviewed in Peters, 2002). Once it has been activated, separase cleaves the cohesin subunit Scc1, which triggers opening of the cohesin ring, dissociation of cohesin complexes from chromosomes, dissolution of sister chromatid cohesion and separation of the two sets of replicated chromosomes to the opposite poles of the mitotic spindle (Tomonaga et al., 2000; Uhlmann et al., 1999; Waizenegger et al., 2000). The concomitant degradation of cyclin B inactivates Cdk1, which leads to cell's exit from mitosis and to the formation of the two daughter cells.

\section{Meiosis}

Meiosis is a specialised form of cell division that produces gametes, which contain either the maternal or the parental copy of each chromosome. Nonetheless, meiosis shares common features with mitosis as well as specific mechanisms. Meiosis involves a single $S$ phase followed by two successive rounds of chromosome segregation without intervening DNA replication, and produces haploid germinal cells. Similar to mitosis, replication of DNA prior meiotic division gives rise to replicated sister chromatids that are linked to each other along their entire length by meiotic cohesin complexes (Figure 3). During prophase I, homologous paternal and maternal chromosomes become attached to each other through a mechanism called homolog pairing or synapsis. This pairing depends on a large protein 
complex called the synaptonemal complex. These homologs pairs (also called tetrads or bivalents) undergo exchanges of DNA segments between paternal and maternal non-sister chromatids by homologous recombination, which give rise to crossover. Once homologous recombination has occurred in late prophase I, the synaptonemal complex is dissolved. Therefore, homologous chromosomes are held together solely by cohesion between sister chromatids distal to the site of recombination. At metaphase I, bivalents are aligned on the meiotic microtubule spindle in a monopolar fashion, that is, with the two kinetochores of each sister chromatid pair facing toward the same spindle pole. At the metaphase I-toanaphase I transition, the meiotic cohesin subunit Rec8 located on the arms of chromosomes is cleaved by separase, allowing the segregation of homologous chromosomes to opposite poles during anaphase I. Thus, the meiosis I gives rise to two cells that only contains either the maternal or the paternal copy of each chromosome, eventually recombined. Due to this peculiarity, meiosis I is also referred to as the reductional division. Central to meiosis is the protection of Rec8 cleavage from separase at centromeric regions by a mechanism that is conserved throughout evolution and that depends on Shugoshin. Unlike in budding yeast and in fruit fly where only one shugoshin protein is found, two shugoshin-like proteins exist in other eukaryotes, and are called Sgo1 and Sgo2. In all eukaryotes cohesin is protected from cleavage by separase in meiosis I, whereas it is protected from non-proteolytic removal in mitosis (Katis et al., 2004; Kitajima et al., 2004; Kitajima et al., 2006; Rabitsch et al., 2004; Riedel et al., 2006). Recent data indicate that Shugoshin-PP2A complex protects Rec8 from cleavage by separase by counteracting its phosphorylation, thereby rendering Rec 8 a poor substrate for separase (Katis et al., 2010). In mouse oocyte, although both Sgo1 and Sgo2 are

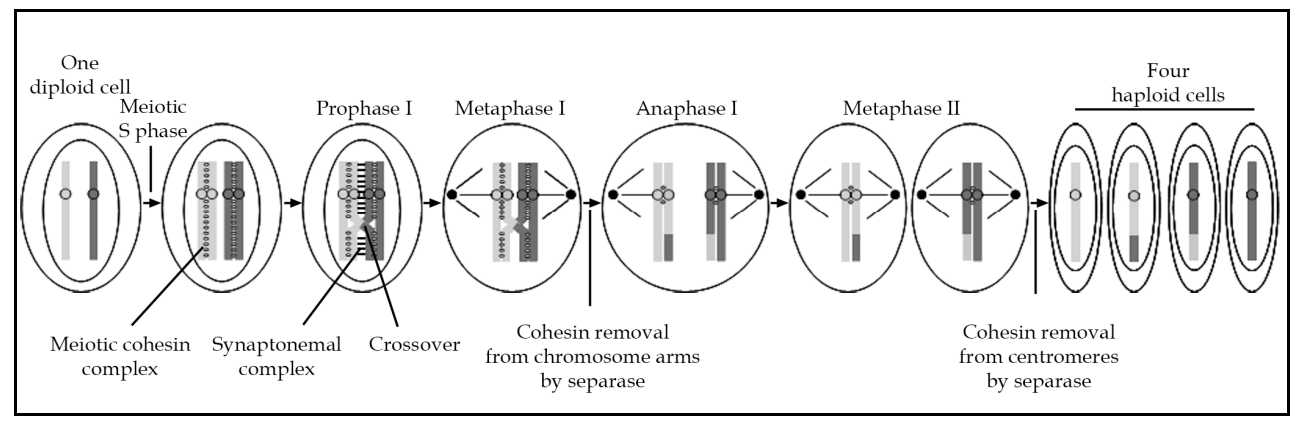

After completion of the meiotic $S$ phase, sister chromatids are held together by meiotic cohesin complexes along their entire length. During prophase I, homologous chromosomes pair and remain associated to each other by the synaptonemal complex, and homologous recombination occurs and results in crossover. Once the synaptonemal complex has been dissolved late in prophase I, homologues remain associated only by cohesin located at distal position from the site of recombination. At metaphase I, homologues are bi-oriented on the first meiotic spindle, with both kinetochores from the same pair of sister chromatids facing the same spindle pole. Active separase cleaves Rec8 from chromosome arms, while cohesin complexes present at centromeres are protected by Shugoshin from cleavage by separase, thereby allowing proper bipolar attachment of sister chromatids on the microtubule spindle at metaphase II. Centromeric Rec 8 is then cleaved by separase, allowing sister chromatid segregation. Once meiosis has been completed, four haploid cells are formed.

Fig. 3. Overview of meiosis 
present in meiotic cells, Sgo2 appears to be the main protector of Rec8 cleavage (Lee et al., 2008; Llano et al., 2008). During meiosis II, also referred to as equational division, sister chromatids align on the second meiotic spindle in a bipolar manner, similar to the situation in mitosis. Once all chromosomes have been properly bi-oriented, separase cleaves centromeric Rec 8 and sister chromatids are segregated between the two future haploid cells. How the function of $\mathrm{Sgo} 2$ in protecting Rec8 from its cleavage by separase is alleviated is still unknown. Once meiosis has been completed, four haploid cells are formed. It has to be noted that during spermatogenesis, four gametes arise from one meiotic division, whereas during oogenesis meiotic divisions are asymmetric and produce only one gamete. Furthermore, vertebrate oocytes are arrested at the metaphase stage of the second meiotic division and the completion of oocyte meiosis is triggered by fertilisation that induces the metaphase II-to-anaphase II transition.

\section{Defective cohesion and aneuploidy}

The sister chromatid cohesion is absolutely essential for the accurate distribution of the replicated genomes during mitosis and meiosis, and therefore, for the faithful transmission of genetic information from one generation of cells or organisms to the next. As a consequence, cohesion defects in somatic cells would lead to unequal distribution of chromosomes that leads to genomic instability and would thereby participate in the appearance of cancers. Similarly, during gametogenesis, defects in cohesion could lead to aneuploid gametes and infertility.

\subsection{Mitosis}

For more than a century, it is well known that genome instability and chromosomal aberrations are associated with cancer. Already in the early $20^{\text {th }}$ century Theodor Boveri proposed that chromosomal instability contributes to cancer development. However, the causal link between aneuploidy and carcinogenesis has remained difficult to be experimentally addressed. Recently, it has been reported that, in yeast cells, aneuploidy induces an increase in DNA recombination and a decrease in DNA damage repair efficiency (Sheltzer et al., 2011), which are two frequent forms of genomic instability that are found in cancer cells. This clearly demonstrates that aneuploidy per se is able to induce chromosomal instability. Thus, defective sister chromatid cohesion can result in chromosome missegregation (Figure 4) that leads to aneuploidy and chromosomal instability, which in fine promotes tumourigenesis. Consistent with the association between an altered sister chromatid cohesion apparatus and tumourigenesis, both mutations and abnormal expression of genes encoding cohesin subunits have been found in various human cancers (for review see Mannini and Musio, 2011; (Xu et al., 2011). For instance, the core cohesin subunits Scc1 and Smc3 are overexpressed in breast and prostate cancers, and in colon carcinomas, respectively. Also, separase is found overexpressed in a variety of cancers including breast cancers (70 \%) and osteosarcomas (for review see Mannini and Musio). More importantly, somatic mutations of Smc1a, Smc3 and STAG3 genes have been found in colorectal cancers (4, 1 and 1 of 130, respectively) (Barber et al., 2008) and mutation of Scc1, SA2, STAG3 were found in lung carcinomas (1, 1 and 1 of 12, respectively) (Xu et al., 2011) and references therein). Furthermore, genes encoding proteins involved in the regulation of cohesin are also found mutated in different types of cancer. In particular, Scc2 gene was 


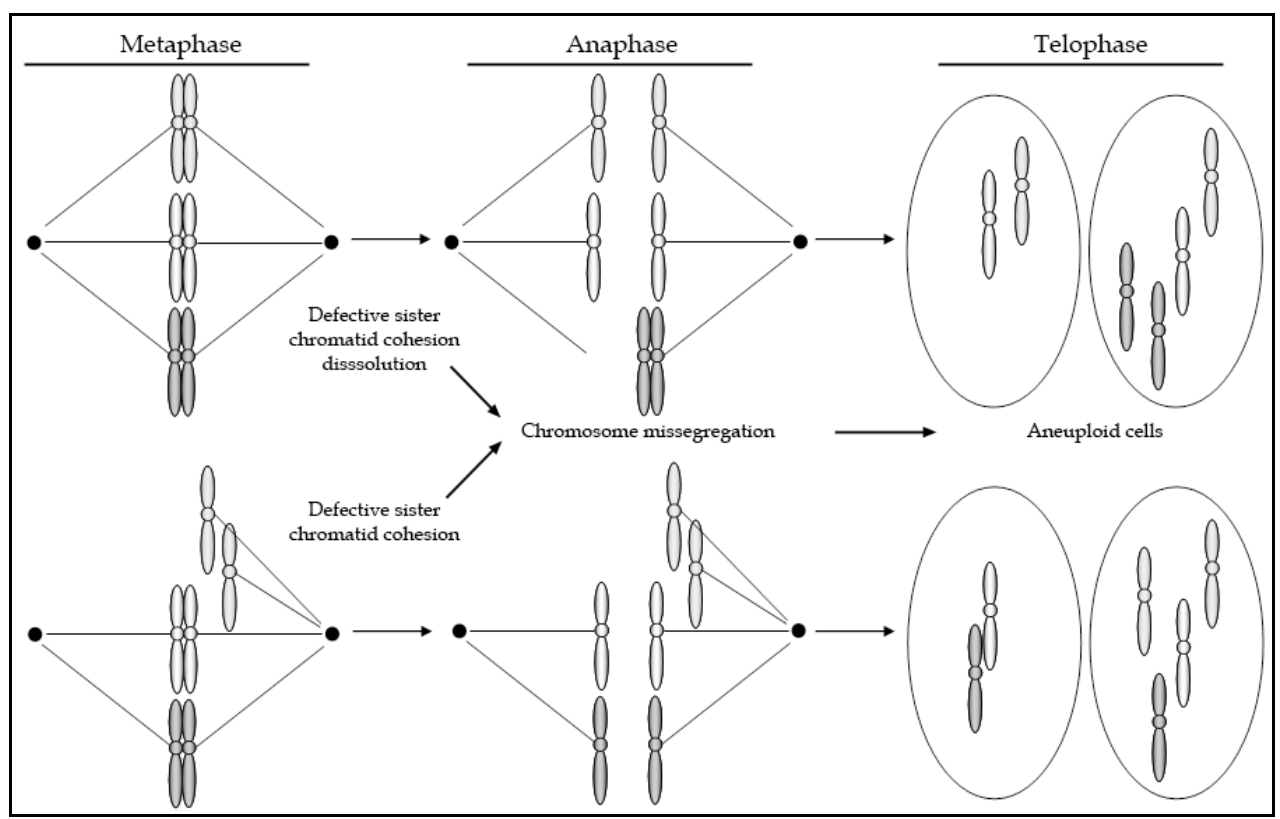

Schematic representation of chromosome missegregation as a cause of aneuploidy in somatic cells. During early mitosis, the spindle microtubule forces align condensed chromosomes on the metaphase plate. At the anaphase, one copy of each chromosome is pulled to one spindle pole. If the cohesin release from chromosomes is altered, sister chromatids would fail to separate and the two sister chromatids would be inherited by one daughter cell. Similarly, the deficient sister chromatid cohesion would lead in the premature separation of sister chromatids. Separated sisters would fail to align on the metaphase plate, which could eventually lead to their co-segregation into one daughter cell.

Fig. 4. Misregulation of sister chromatid cohesion leads to aneuploidy

shown to carry mutations in breast (1 of 48), lung ( 2 of 11) and kidney (1 of 101) carcinomas as well as in colorectal cancers (4 of 130), whereas mutations in separase gene were identified in kidney ( 1 of 101) and lung (1 of 12) carcinomas (Xu et al., 2011). Altogether, these observations underline the strong association that exists between altered sister chromatid cohesion apparatus and cancers. The direct implication of cohesin mutations in aneuploidy and chromosomal instability has been directly addressed recently (Solomon et al., 2011). The authors used single-nucleotide polymorphism analysis to characterise chromosomal rearrangements in human glioblastoma cell lines and thereby identified a genomic deletion of the $X$ chromosome that contains the cohesin subunit SA2 gene. Accordingly, no expression of SA2 could be observed in these cell lines. Absence of functional SA2 protein expression was extended to others genomic rearrangement (frameshift insertions and deletions, mutations of splicing regulatory elements) in other cancer cell lines (Solomon et al., 2011). Then, the authors addressed whether SA2 gene mutations induce genomic instability and aneuploidy by correcting endogenous locus using gene targeting in glioblastoma cell lines. Non-corrected cell lines exhibited premature separation of sister chromatids similar to the phenotype observed when cohesin is inactivated. Quite 
remarkably, the correction of SA2 was sufficient to rescue the loss-of-cohesion phenotype in this cancer cell line, demonstrating that SA2 mutation were the cause of the observed defective cohesion. Furthermore, the authors showed that the correction of SA2 not only rescues sister chromatid cohesion, but also reduces the number of defective mitotic divisions and the chromosome missegregation at anaphase. Finally, restoring SA2 expression also triggered a reduction in the variability in chromosome numbers between independent cells, indicative of an increase in genome stability. Altogether these works demonstrate that alteration of sister chromatid cohesion apparatus leads to missegregation of chromosomes and to aneuploidy and chromosome instability. This indicates that the function of the cohesin complex in maintaining the sister chromatid cohesion acts as an important barrier against tumorigenesis. Whether other known functions of cohesin, i.e. in the DNA damage response or in gene expression, also participate in carcinogenesis still needs to be uncovered.

\subsection{Meiosis}

The proper segregation of chromosomes during meiosis is of crucial importance in all living organisms, as aneuploidy leads to embryonic death and severe disorders, such as infertility and Down syndrome. Recent efforts from the scientific community have highlighted the importance of cohesin in preventing aneuploidy during meiosis in mammals. In human, oocytes wait up to several decades until they finally resume the first meiotic division, and it is well established that oocyte aneuploidy increases with age. Accordingly, age-related aneuploidy in oocyte correlates with a dramatic increase of trisomic pregnancy. Aneuploidy commonly arises from segregation errors during oocyte meiosis I, and relates to the long arrest of oocytes in preceding stages of meiosis. In these oocytes, the cohesion between chromosomes must be maintained for an extraordinary long period of time (up to $\sim$ fifty years in human), and depends on cohesin complexes. Recent studies in mouse have suggested that the loss of sister chromatid cohesion is a leading cause of aneuploidy in oocyte, and is due to a progressive deterioration of cohesin itself. Indeed, these studies have reported that the meiotic cohesin subunit Rec8 (Chiang et al., 2010; Lister et al., 2010) and the protein Sgo2 that protects centromeric cohesin in meiosis I (Lister et al., 2010) decrease on meiotic chromosomes with age. The degradation of cohesin correlates with an increased distance between sister kinetochores and with the loss of sister chromatid cohesion (Chiang et al., 2010; Lister et al., 2010). Altogether, these data indicate that cohesin is essential to maintain sister chromatid cohesion in arrested oocytes, and highlight the importance of the cohesin function in preventing aneuploidy and infertility in mammalian oocytes. Determining how cohesin gets degraded, if and how it can be prevented would be of great interest in the future.

\section{Conclusion}

The sister chromatid cohesion apparatus protects somatic and germinal cells from unequal chromosome segregation and aneuploidy. Recent advances in the field have pointed to the misregulation of cohesion factors, mainly cohesin, as a cause for aneuploidy-based human disorders from cancer to trisomies and infertility. In the future, we will have to decipher the molecular mechanisms of both normal and defective sister chromatid cohesion, as well as its links with human pathologies, including cancers. Furthermore, it will be interesting to address whether one could take advantage of our knowledge on the molecular function of cohesin to develop new approaches to treat cancer cells. Finally, it is of great importance to 
determine if, and to which extent, the other known functions of cohesin and its associated factors, namely regulation of gene expression and the DNA damage response, also participate in tumourigenesis.

\section{Further reading}

In this chapter, we aimed at presenting the basic current knowledge about the sister chromatid cohesion apparatus, its role and regulation in the segregation of replicated chromosomes during mitosis and meiosis, and how cohesion defects lead to aneuploidy. For those colleagues who wish to learn more about particular aspects of cohesin in chromosome biology, there are some dedicated reviews that we shall recommend: cohesin mechanistic and its regulation (Nasmyth and Haering, 2009; Onn et al., 2008; Peters et al., 2008), cohesin in gene regulation (Dorsett, 2007; Wendt and Peters, 2009), DNA damage response (Strom and Sjogren, 2007; Watrin and Peters, 2006), meiosis and gametogenesis (Jessberger, 2003; Petronczki et al., 2003), aneuploidy and cancer (Barbero, 2011; Nicholson and Cimini, 2011).

\section{Acknowledgment}

We are grateful to Jacek Kubiak for his comments on the manuscript. Work in Claude Prigent 's lab is funded by the Centre National de la Recherche Scientifique (CNRS), the University of Rennes I, and by grants from the Agence Nationale pour la Recherche, the Association pour la Recherche contre le Cancer and the Ligue Nationale contre le Cancer.

\section{References}

Anderson, D.E., Losada, A., Erickson, H.P. and Hirano, T. (2002) Condensin and cohesin display different arm conformations with characteristic hinge angles. J Cell Biol, 156, 419-424.

Arumugam, P., Gruber, S., Tanaka, K., Haering, C.H., Mechtler, K. and Nasmyth, K. (2003) ATP hydrolysis is required for cohesin's association with chromosomes. Curr Biol, 13, 1941-1953.

Barber, T.D., McManus, K., Yuen, K.W., Reis, M., Parmigiani, G., Shen, D., Barrett, I., Nouhi, Y., Spencer, F., Markowitz, S., Velculescu, V.E., Kinzler, K.W., Vogelstein, B., Lengauer, C. and Hieter, P. (2008) Chromatid cohesion defects may underlie chromosome instability in human colorectal cancers. Proc Natl Acad Sci U S A, 105, 3443-3448.

Barbero, J.L. (2011) Sister chromatid cohesion control and aneuploidy. Cytogenet Genome Res, $133,223-233$.

Bernard, P., Drogat, J., Maure, J.F., Dheur, S., Vaur, S., Genier, S. and Javerzat, J.P. (2006) A screen for cohesion mutants uncovers Ssl3, the fission yeast counterpart of the cohesin loading factor Scc4. Curr Biol., 16, 875-881.

Canudas, S. and Smith, S. (2009) Differential regulation of telomere and centromere cohesion by the Scc3 homologues SA1 and SA2, respectively, in human cells. J Cell Biol, 187, 165-173.

Chiang, T., Duncan, F.E., Schindler, K., Schultz, R.M. and Lampson, M.A. (2010) Evidence that weakened centromere cohesion is a leading cause of age-related aneuploidy in oocytes. Curr Biol, 20, 1522-1528. 
Ciosk, R., Shirayama, M., Shevchenko, A., Tanaka, T., Toth, A. and Nasmyth, K. (2000) Cohesin's binding to chromosomes depends on a separate complex consisting of Scc2 and Scc4 proteins. Mol Cell, 5, 243-254.

Dai, J., Sullivan, B.A. and Higgins, J.M. (2006) Regulation of mitotic chromosome cohesion by Haspin and Aurora B. Dev Cell, 11, 741-750.

Dorsett, D. (2007) Roles of the sister chromatid cohesion apparatus in gene expression, development, and human syndromes. Chromosoma., 116, 1-13. Epub 2006 Jul 2004.

Dorsett, D., Eissenberg, J.C., Misulovin, Z., Martens, A., Redding, B. and McKim, K. (2005) Effects of sister chromatid cohesion proteins on cut gene expression during wing development in Drosophila. Development, 5, 5.

Feytout, A., Vaur, S., Genier, S., Vazquez, S. and Javerzat, J.P. (2011) Psm3 acetylation on conserved lysine residues is dispensable for viability in fission yeast but contributes to Eso1-mediated sister chromatid cohesion by antagonizing Wpl1. Mol Cell Biol, 31, 1771-1786.

Furuya, K., Takahashi, K. and Yanagida, M. (1998) Faithful anaphase is ensured by Mis4, a sister chromatid cohesion molecule required in $\mathrm{S}$ phase and not destroyed in G1 phase. Genes Dev, 12, 3408-3418.

Gandhi, R., Gillespie, P.J. and Hirano, T. (2006) Human Wapl is a cohesin-binding protein that promotes sister-chromatid resolution in mitotic prophase. Curr Biol., 16, 24062417. Epub 2006 Nov 2416.

Gerlich, D., Koch, B., Dupeux, F., Peters, J.M. and Ellenberg, J. (2006) Live-cell imaging reveals a stable cohesin-chromatin interaction after but not before DNA replication. Curr Biol, 16, 1571-1578.

Gillespie, P.J. and Hirano, T. (2004) Scc2 couples replication licensing to sister chromatid cohesion in Xenopus egg extracts. Curr Biol, 14, 1598-1603.

Gruber, S., Arumugam, P., Katou, Y., Kuglitsch, D., Helmhart, W., Shirahige, K. and Nasmyth, K. (2006) Evidence that loading of cohesin onto chromosomes involves opening of its SMC hinge. Cell, 127, 523-537.

Gruber, S., Haering, C.H. and Nasmyth, K. (2003) Chromosomal cohesin forms a ring. Cell, $112,765-777$.

Haering, C.H., Lowe, J., Hochwagen, A. and Nasmyth, K. (2002) Molecular architecture of SMC proteins and the yeast cohesin complex. Mol Cell, 9, 773-788.

Haering, C.H., Schoffnegger, D., Nishino, T., Helmhart, W., Nasmyth, K. and Lowe, J. (2004) Structure and stability of cohesin's Smc1-kleisin interaction. Mol Cell., 15, 951-964.

Hartman, T., Stead, K., Koshland, D. and Guacci, V. (2000) Pds5p is an essential chromosomal protein required for both sister chromatid cohesion and condensation in Saccharomyces cerevisiae. J Cell Biol, 151, 613-626.

Hauf, S., Roitinger, E., Koch, B., Dittrich, C.M., Mechtler, K. and Peters, J.M. (2005) Dissociation of cohesin from chromosome arms and loss of arm cohesion during early mitosis depends on phosphorylation of SA2. PLoS Biol, 3, e69. Epub 2005 Mar 2001.

Hirota, T., Gerlich, D., Koch, B., Ellenberg, J. and Peters, J.M. (2004) Distinct functions of condensin I and II in mitotic chromosome assembly. J Cell Sci, 117, 6435-6445. Epub 2004 Nov 6430.

Hou, F. and Zou, H. (2005) Two human orthologues of Eco1/Ctf7 acetyltransferases are both required for proper sister-chromatid cohesion. Mol Biol Cell., 16, 3908-3918. Epub 2005 Jun 3915.

Huang, C.E., Milutinovich, M. and Koshland, D. (2005) Rings, bracelet or snaps: fashionable alternatives for Smc complexes. Philos Trans R Soc Lond B Biol Sci, 360, 537-542. 
Jessberger, R. (2003) SMC proteins at the crossroads of diverse chromosomal processes. IUBMB Life, 55, 643-652.

Katis, V.L., Galova, M., Rabitsch, K.P., Gregan, J. and Nasmyth, K. (2004) Maintenance of cohesin at centromeres after meiosis I in budding yeast requires a kinetochoreassociated protein related to MEI-S332. Curr Biol, 14, 560-572.

Katis, V.L., Lipp, J.J., Imre, R., Bogdanova, A., Okaz, E., Habermann, B., Mechtler, K., Nasmyth, K. and Zachariae, W. (2010) Rec8 phosphorylation by casein kinase 1 and Cdc7-Dbf4 kinase regulates cohesin cleavage by separase during meiosis. Dev Cell, 18, 397-409.

Kawashima, S.A., Yamagishi, Y., Honda, T., Ishiguro, K. and Watanabe, Y. (2010) Phosphorylation of $\mathrm{H} 2 \mathrm{~A}$ by Bub1 prevents chromosomal instability through localizing shugoshin. Science, 327, 172-177.

Kim, B.J., Li, Y., Zhang, J., Xi, Y., Li, Y., Yang, T., Jung, T., Jung, S.Y., Pan, X., Chen, R., Li, W., Wang, Y. and Qin, J. (2010) Genome-wide reinforcement of cohesin binding at pre-existing cohesin sites in response to ionizing radiation in human cells. J Biol Chem, 285, 22784-22792.

Kitajima, T.S., Kawashima, S.A. and Watanabe, Y. (2004) The conserved kinetochore protein shugoshin protects centromeric cohesion during meiosis. Nature, 427, 510-517. Epub 2004 Jan 2018.

Kitajima, T.S., Sakuno, T., Ishiguro, K., Iemura, S., Natsume, T., Kawashima, S.A. and Watanabe, Y. (2006) Shugoshin collaborates with protein phosphatase 2A to protect cohesin. Nature, 441, 46-52.

Kueng, S., Hegemann, B., Peters, B.H., Lipp, J.J., Schleiffer, A., Mechtler, K. and Peters, J.M. (2006) Wapl controls the dynamic association of cohesin with chromatin. Cell., 127, 955-967. Epub 2006 Nov 2016.

Lee, J., Kitajima, T.S., Tanno, Y., Yoshida, K., Morita, T., Miyano, T., Miyake, M. and Watanabe, Y. (2008) Unified mode of centromeric protection by shugoshin in mammalian oocytes and somatic cells. Nat Cell Biol, 10, 42-52.

Lenart, P., Petronczki, M., Steegmaier, M., Di Fiore, B., Lipp, J.J., Hoffmann, M., Rettig, W.J., Kraut, N. and Peters, J.M. (2007) The small-molecule inhibitor BI 2536 reveals novel insights into mitotic roles of polo-like kinase 1. Curr Biol., 17, 304-315. Epub 2007 Feb 2008.

Lister, L.M., Kouznetsova, A., Hyslop, L.A., Kalleas, D., Pace, S.L., Barel, J.C., Nathan, A., Floros, V., Adelfalk, C., Watanabe, Y., Jessberger, R., Kirkwood, T.B., Hoog, C. and Herbert, M. (2010) Age-related meiotic segregation errors in mammalian oocytes are preceded by depletion of cohesin and Sgo2. Curr Biol, 20, 1511-1521.

Llano, E., Gomez, R., Gutierrez-Caballero, C., Herran, Y., Sanchez-Martin, M., VazquezQuinones, L., Hernandez, T., de Alava, E., Cuadrado, A., Barbero, J.L., Suja, J.A. and Pendas, A.M. (2008) Shugoshin-2 is essential for the completion of meiosis but not for mitotic cell division in mice. Genes Dev, 22, 2400-2413.

Losada, A., Hirano, M. and Hirano, T. (1998) Identification of Xenopus SMC protein complexes required for sister chromatid cohesion. Genes Dev, 12, 1986-1997.

Losada, A., Hirano, M. and Hirano, T. (2002) Cohesin release is required for sister chromatid resolution, but not for condensin-mediated compaction, at the onset of mitosis. Genes Dev, 16, 3004-3016.

Losada, A., Yokochi, T., Kobayashi, R. and Hirano, T. (2000) Identification and Characterization of SA/Scc3p Subunits in the Xenopus and Human Cohesin Complexes. J Cell Biol, 150, 405-416. 
Mc Intyre, J., Muller, E.G., Weitzer, S., Snydsman, B.E., Davis, T.N. and Uhlmann, F. (2007) In vivo analysis of cohesin architecture using FRET in the budding yeast Saccharomyces cerevisiae. Embo J, 26, 3783-3793.

McGuinness, B.E., Hirota, T., Kudo, N.R., Peters, J.M. and Nasmyth, K. (2005) Shugoshin prevents dissociation of cohesin from centromeres during mitosis in vertebrate cells. PLoS Biol, 3, e86. Epub 2005 Mar 2001.

Melby, T.E., Ciampaglio, C.N., Briscoe, G. and Erickson, H.P. (1998) The symmetrical structure of structural maintenance of chromosomes (SMC) and MukB proteins: long, antiparallel coiled coils, folded at a flexible hinge. J Cell Biol, 142, 1595-1604.

Michaelis, C., Ciosk, R. and Nasmyth, K. (1997) Cohesins: chromosomal proteins that prevent premature separation of sister chromatids. Cell, 91, 35-45.

Milutinovich, M., Unal, E., Ward, C., Skibbens, R.V. and Koshland, D. (2007) A multi-step pathway for the establishment of sister chromatid cohesion. PLoS Genet., 3, e12. Epub 2006 Dec 2008.

Nasmyth, K. (2001) Disseminating the genome: joining, resolving, and separating sister chromatids during mitosis and meiosis. Annu Rev Genet, 35, 673-745.

Nasmyth, K. and Haering, C.H. (2009) Cohesin: its roles and mechanisms. Annu Rev Genet, $43,525-558$.

Nicholson, J.M. and Cimini, D. (2011) How mitotic errors contribute to karyotypic diversity in cancer. Adv Cancer Res, 112, 43-75.

Nishiyama, T., Ladurner, R., Schmitz, J., Kreidl, E., Schleiffer, A., Bhaskara, V., Bando, M., Shirahige, K., Hyman, A.A., Mechtler, K. and Peters, J.M. (2010) Sororin mediates sister chromatid cohesion by antagonizing Wapl. Cell, 143, 737-749.

Onn, I., Heidinger-Pauli, J.M., Guacci, V., Unal, E. and Koshland, D.E. (2008) Sister chromatid cohesion: a simple concept with a complex reality. Annu Rev Cell Dev Biol, 24, 105-129.

Panizza, S., Tanaka, T., Hochwagen, A., Eisenhaber, F. and Nasmyth, K. (2000) Pds5 cooperates with cohesin in maintaining sister chromatid cohesion. Curr Biol, 10, 1557-1564.

Peters, J.M. (2002) The anaphase-promoting complex: proteolysis in mitosis and beyond. Mol Cell, 9, 931-943.

Peters, J.M., Tedeschi, A. and Schmitz, J. (2008) The cohesin complex and its roles in chromosome biology. Genes Dev, 22, 3089-3114.

Petronczki, M., Siomos, M.F. and Nasmyth, K. (2003) Un menage a quatre: the molecular biology of chromosome segregation in meiosis. Cell, 112, 423-440.

Rabitsch, K.P., Gregan, J., Schleiffer, A., Javerzat, J.P., Eisenhaber, F. and Nasmyth, K. (2004) Two fission yeast homologs of Drosophila Mei-S332 are required for chromosome segregation during meiosis I and II. Curr Biol, 14, 287-301.

Rankin, S., Ayad, N.G. and Kirschner, M.W. (2005) Sororin, a substrate of the anaphasepromoting complex, is required for sister chromatid cohesion in vertebrates. Mol Cell, 18, 185-200.

Riedel, C.G., Katis, V.L., Katou, Y., Mori, S., Itoh, T., Helmhart, W., Galova, M., Petronczki, M., Gregan, J., Cetin, B., Mudrak, I., Ogris, E., Mechtler, K., Pelletier, L., Buchholz, F., Shirahige, K. and Nasmyth, K. (2006) Protein phosphatase 2A protects centromeric sister chromatid cohesion during meiosis I. Nature, 441, 53-61.

Rolef Ben-Shahar, T., Heeger, S., Lehane, C., East, P., Flynn, H., Skehel, M. and Uhlmann, F. (2008) Eco1-dependent cohesin acetylation during establishment of sister chromatid cohesion. Science, 321, 563-566. 
Rollins, R.A., Korom, M., Aulner, N., Martens, A. and Dorsett, D. (2004) Drosophila nipped-B protein supports sister chromatid cohesion and opposes the stromalin/Scc3 cohesion factor to facilitate long-range activation of the cut gene. Mol Cell Biol, 24, 3100-3111.

Rowland, B.D., Roig, M.B., Nishino, T., Kurze, A., Uluocak, P., Mishra, A., Beckouet, F., Underwood, P., Metson, J., Imre, R., Mechtler, K., Katis, V.L. and Nasmyth, K. (2009) Building sister chromatid cohesion: smc3 acetylation counteracts an antiestablishment activity. Mol Cell, 33, 763-774.

Salic, A., Waters, J.C. and Mitchison, T.J. (2004) Vertebrate shugoshin links sister centromere cohesion and kinetochore microtubule stability in mitosis. Cell, 118, 567-578.

Schmitz, J., Watrin, E., Lenart, P., Mechtler, K. and Peters, J.M. (2007) Sororin is required for stable binding of cohesin to chromatin and for sister chromatid cohesion in interphase. Curr Biol, 17, 630-636.

Sheltzer, J.M., Blank, H.M., Pfau, S.J., Tange, Y., George, B.M., Humpton, T.J., Brito, I.L., Hiraoka, Y., Niwa, O. and Amon, A. (2011) Aneuploidy drives genomic instability in yeast. Science, 333, 1026-1030.

Shintomi, K. and Hirano, T. (2009) Releasing cohesin from chromosome arms in early mitosis: opposing actions of Wapl-Pds5 and Sgo1. Genes Dev, 23, 2224-2236.

Sjogren, C. and Nasmyth, K. (2001) Sister chromatid cohesion is required for postreplicative double-strand break repair in Saccharomyces cerevisiae. Curr Biol, 11, 991-995.

Skibbens, R.V., Corson, L.B., Koshland, D. and Hieter, P. (1999) Ctf7p is essential for sister chromatid cohesion and links mitotic chromosome structure to the DNA replication machinery. Genes Dev, 13, 307-319.

Solomon, D.A., Kim, T., Diaz-Martinez, L.A., Fair, J., Elkahloun, A.G., Harris, B.T., Toretsky, J.A., Rosenberg, S.A., Shukla, N., Ladanyi, M., Samuels, Y., James, C.D., Yu, H., Kim, J.S. and Waldman, T. (2011) Mutational inactivation of STAG2 causes aneuploidy in human cancer. Science, 333, 1039-1043.

Strom, L., Karlsson, C., Lindroos, H.B., Wedahl, S., Katou, Y., Shirahige, K. and Sjogren, C. (2007) Postreplicative formation of cohesion is required for repair and induced by a single DNA break. Science., 317, 242-245.

Strom, L. and Sjogren, C. (2007) Chromosome segregation and double-strand break repair a complex connection. Curr Opin Cell Biol., 19, 344-349. Epub 2007 Apr 2026.

Strunnikov, A.V., Larionov, V.L. and Koshland, D. (1993) SMC1: an essential yeast gene encoding a putative head-rod-tail protein is required for nuclear division and defines a new ubiquitous protein family. J Cell Biol, 123, 1635-1648.

Sumara, I., Vorlaufer, E., Gieffers, C., Peters, B.H. and Peters, J.M. (2000) Characterization of vertebrate cohesin complexes and their regulation in prophase. J Cell Biol, 151, 749-762.

Sumara, I., Vorlaufer, E., Stukenberg, P.T., Kelm, O., Redemann, N., Nigg, E.A. and Peters, J.M. (2002) The dissociation of cohesin from chromosomes in prophase is regulated by Polo-like kinase. Mol Cell, 9, 515-525.

Sutani, T., Kawaguchi, T., Kanno, R., Itoh, T. and Shirahige, K. (2009) Budding yeast Wpl1(Rad61)-Pds5 complex counteracts sister chromatid cohesion-establishing reaction. Curr Biol, 19, 492-497.

Takahashi, T.S., Yiu, P., Chou, M.F., Gygi, S. and Walter, J.C. (2004) Recruitment of Xenopus Scc2 and cohesin to chromatin requires the pre-replication complex. Nat Cell Biol, 6, 991-996. Epub 2004 Sep 2026.

Tanaka, K., Hao, Z., Kai, M. and Okayama, H. (2001) Establishment and maintenance of sister chromatid cohesion in fission yeast by a unique mechanism. Embo J, 20, 5779-5790. 
Tang, Z., Sun, Y., Harley, S.E., Zou, H. and Yu, H. (2004) Human Bub1 protects centromeric sister-chromatid cohesion through Shugoshin during mitosis. Proc Natl Acad Sci U S A, 101, 18012-18017. Epub 12004 Dec 18016.

Tomonaga, T., Nagao, K., Kawasaki, Y., Furuya, K., Murakami, A., Morishita, J., Yuasa, T., Sutani, T., Kearsey, S.E., Uhlmann, F., Nasmyth, K. and Yanagida, M. (2000) Characterization of fission yeast cohesin: essential anaphase proteolysis of $\operatorname{Rad} 21$ phosphorylated in the S phase. Genes Dev, 14, 2757-2770.

Toth, A., Ciosk, R., Uhlmann, F., Galova, M., Schleiffer, A. and Nasmyth, K. (1999) Yeast cohesin complex requires a conserved protein, Eco1p(Ctf7), to establish cohesion between sister chromatids during DNA replication. Genes Dev, 13, 320-333.

Uhlmann, F., Lottspeich, F. and Nasmyth, K. (1999) Sister-chromatid separation at anaphase onset is promoted by cleavage of the cohesin subunit Scc1. Nature, 400, 37-42.

Uhlmann, F. and Nasmyth, K. (1998) Cohesion between sister chromatids must be established during DNA replication. Curr Biol, 8, 1095-1101.

Unal, E., Heidinger-Pauli, J.M., Kim, W., Guacci, V., Onn, I., Gygi, S.P. and Koshland, D.E. (2008) A molecular determinant for the establishment of sister chromatid cohesion. Science, 321, 566-569.

Unal, E., Heidinger-Pauli, J.M. and Koshland, D. (2007) DNA double-strand breaks trigger genome-wide sister-chromatid cohesion through Eco1 (Ctf7). Science., 317, 245-248.

Verni, F., Gandhi, R., Goldberg, M.L. and Gatti, M. (2000) Genetic and molecular analysis of wings apart-like (wapl), a gene controlling heterochromatin organization in Drosophila melanogaster. Genetics, 154, 1693-1710.

Waizenegger, I.C., Hauf, S., Meinke, A. and Peters, J.M. (2000) Two distinct pathways remove mammalian cohesin from chromosome arms in prophase and from centromeres in anaphase. Cell, 103, 399-410.

Wang, S.W., Read, R.L. and Norbury, C.J. (2002) Fission yeast Pds5 is required for accurate chromosome segregation and for survival after DNA damage or metaphase arrest. J Cell Sci, 115, 587-598.

Watrin, E. and Peters, J.M. (2006) Cohesin and DNA damage repair. Exp Cell Res., 312, 26872693. Epub 2006 Jun 2622.

Watrin, E., Schleiffer, A., Tanaka, K., Eisenhaber, F., Nasmyth, K. and Peters, J.M. (2006) Human scc4 is required for cohesin binding to chromatin, sister-chromatid cohesion, and mitotic progression. Curr Biol., 16, 863-874.

Weitzer, S., Lehane, C. and Uhlmann, F. (2003) A model for ATP hydrolysis-dependent binding of cohesin to DNA. Curr Biol, 13, 1930-1940.

Wendt, K.S. and Peters, J.M. (2009) How cohesin and CTCF cooperate in regulating gene expression. Chromosome Res, 17, 201-214.

Xu, H., Tomaszewski, J.M. and McKay, M.J. (2011) Can corruption of chromosome cohesion create a conduit to cancer? Nat Rev Cancer, 11, 199-210.

Zhang, J., Shi, X., Li, Y., Kim, B.J., Jia, J., Huang, Z., Yang, T., Fu, X., Jung, S.Y., Wang, Y., Zhang, P., Kim, S.T., Pan, X. and Qin, J. (2008a) Acetylation of Smc3 by Eco1 is required for S phase sister chromatid cohesion in both human and yeast. Mol Cell, 31, 143-151.

Zhang, N., Kuznetsov, S.G., Sharan, S.K., Li, K., Rao, P.H. and Pati, D. (2008b) A handcuff model for the cohesin complex. J Cell Biol, 183, 1019-1031. 


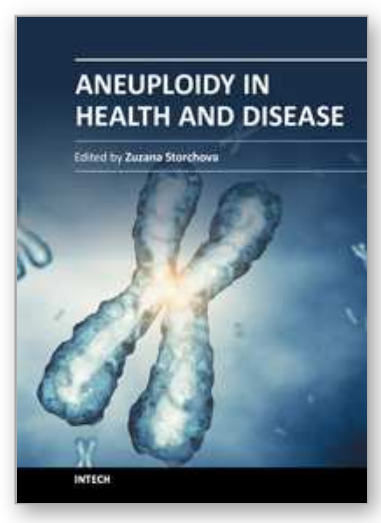

\author{
Aneuploidy in Health and Disease \\ Edited by Dr Zuzana Storchova
}

ISBN 978-953-51-0608-1

Hard cover, 244 pages

Publisher InTech

Published online 16, May, 2012

Published in print edition May, 2012

Aneuploidy means any karyotype that is not euploid, anything that stands outside the norm. Two particular characteristics make the research of aneuploidy challenging. First, it is often hard to distinguish what is a cause and what is a consequence. Secondly, aneuploidy is often associated with a persistent defect in maintenance of genome stability. Thus, working with aneuploid, unstable cells means analyzing an ever changing creature and capturing the features that persist. In the book Aneuploidy in Health and Disease we summarize the recent advances in understanding the causes and consequences of aneuploidy and its link to human pathologies.

\title{
How to reference
}

In order to correctly reference this scholarly work, feel free to copy and paste the following:

Erwan Watrin and Claude Prigent (2012). Sister Chromatid Cohesion and Aneuploidy, Aneuploidy in Health and Disease, Dr Zuzana Storchova (Ed.), ISBN: 978-953-51-0608-1, InTech, Available from:

http://www.intechopen.com/books/aneuploidy-in-health-and-disease/sister-chromatid-cohesion-and-aneuploidy

\section{INTECH}

open science | open minds

\author{
InTech Europe \\ University Campus STeP Ri \\ Slavka Krautzeka 83/A \\ 51000 Rijeka, Croatia \\ Phone: +385 (51) 770447 \\ Fax: +385 (51) 686166 \\ www.intechopen.com
}

\author{
InTech China \\ Unit 405, Office Block, Hotel Equatorial Shanghai \\ No.65, Yan An Road (West), Shanghai, 200040, China \\ 中国上海市延安西路65号上海国际贵都大饭店办公楼 405 单元 \\ Phone: +86-21-62489820 \\ Fax: $+86-21-62489821$
}


(C) 2012 The Author(s). Licensee IntechOpen. This is an open access article distributed under the terms of the Creative Commons Attribution 3.0 License, which permits unrestricted use, distribution, and reproduction in any medium, provided the original work is properly cited. 